\title{
ANALYSIS OF STRUCTURAL CHANGES IN THE ZONES OF LOCAL CATASTROPHIC DESTRUCTION OF PIPELINE SYSTEMS OF OIL REFINING
}

\author{
Murav'ev V.I., Dr. Sci. (Eng.), Professor, Chief Researcher \\ (vmuravyev@mail.ru) \\ Lonchakov S.Z., Cand. Sci. (Eng.), Senior Researcher \\ Pitsyk V.S, Postgraduate
}

Komsomolsk-on-Amur State Technical University (27, pr. Lenina, Komsomolsk-on-Amur, 681031, Russia)

Abstract. It has been found that the most dangerous corrosion type of pipeline systems of oil refining is a pitting one. Based on the volume and thickness measurements, as well as the results of the coercive force measurements, the mechanism of local catastrophic corrosion of pipeline systems of oil refining has been established. A method of nondestructive quality control of welding and subsequent heat treatment of pipeline systems of oil refining has been offered. It will prevent the emergency situations.

Keywords: corrosion, micro- and macro-structure, cavitation, thickness measurements, coercive force, sulfide deposits.

\section{REFERENCES}

1. Luk’yanov S.I., Butin A.V., Evstigneev A.A., Murav'ev V.I., Kim V.A. Magnetic identification of the material microstruc- ture. Kontrol' $i$ diagnostika. 2010, no. 10, pp. 30-33. (In Russ.).

2. Kolotyrkin Ya.M. Metall i korroziya [Metal and corrosion]. Moscow: Metallurgiya, 1985. 88 p. (In Russ.).

3. Murav'evV.I., Bakhmatov P.V, Frolov A.V., Evstigneev A.A., Butin A.V. The impact of structural changes of metal pipes for damage to pipeline systems for petroleum refining. Khimicheskoe i neftegazovoe mashinostroenie. 2011, no. 9, pp. 42-46. (In Russ.).

4. Murav'ev V.I., Evstigneev A.A., Bahmatov P.V., Butin A.V. Analytical evaluation and development of methods to improve the corrosion resistance of structural steels TSPN. Uchenye zapiski «Komsomol'skogo-na-Amure gosudarstvennogo tehnicheskogo universiteta». 2011, no. IV-1 (8), pp. 58-70. (In Russ.).

5. Kim V.A., Murav'ev V.I., Luk'yanov S.I., Butin A.V. Corrosion processes and the resource of technological pipelines of ELOUAVT-3. Khimicheskoe i neftegazovoe mashinostroenie. 2012, no. 11, pp. 35-38. (In Russ.).

6. Uling G.G., Revi R.U. Korroziya i bor'ba s nei. Vvedenie v korrozionnuyu nauku i tekhniku [Corrosion and the fight against it. Introduction to corrosion science and technology]. Sukhotina A.M. ed. Leningrad: Khimiya, 1989. 456 p. (In Russ.).

Received December 24, 2013

\section{МИКРОСТРУКТУРА, ПОЛЯ ВНУТРЕННИХ НАПРЯЖЕНИЙ И АКУСТИЧЕСКИЕ ХАРАКТЕРИСТИКИ МЕТАЛЛА РАЗРУШЕННОГО РОТОРА ПАРОВОЙ ТУРБИНЫ ${ }^{*}$}

\author{
Смирнов А.H. ${ }^{1}$, д.m.н., професcop (galvas .kem@gmai l .com) \\ Абабков Н.В. ${ }^{1}$, к.т.н., доцент \\ Козлов Э.В. ${ }^{2}$, д.ф.-м.н., профессор, заведуюший кафедрой \\ Конева Н.А. ${ }^{2}$, д.ф.-м.н., профессор \\ Быкова Н.В. ${ }^{1}$, инженер научно-инновачионного управления \\ ${ }^{1}$ Кузбасский государственный технический университет им. Т.Ф. Горбачева \\ (Россия, 650000, Кемерово, ул. Весенняя, 28) \\ 2 Томский государственный архитектурно-строительный университет
}

(Россия, 634003, Томск, пл. Соляная, 2)

\begin{abstract}
Аннотация. Проведено исследование металла фрагмента разрушенного ротора паровой турбины разрушающими и неразрушающими методами контроля для выявления причин разрушений и для предотвращения в будущем подобных случаев. Исследовано структурное состояние металла разрушенного ротора с применением методов электронной микроскопии. Установлена взаимосвязь акустических характеристик и параметров микроструктуры металла разрушенного ротора.
\end{abstract}

Ключевые слова: ротор паровой турбины, микроструктура, микротрещины, диагностика.

В настоящее время в России более $80 \%$ (в энергетике более $90 \%$ промышленного оборудования отра- ботало свой расчетный срок. Разрушения технических устройств или их элементов могут привести и приводят

\footnotetext{
* Авторы признательны сотрудникам кафедры физики ТГАСУ Н.А. Поповой, М.В. Федорищевой, Н.Р. Сизоненко, Е.Л. Никоненко и М.П. Калашникову за участие в проведении исследований, а также Б.Р. Фенстеру (ООО»Энергопром - М) за участие в постановочных экспериментах.
} 
к крупным техногенным катастрофам с человеческими жертвами, поэтому особенно актуальной является задача обеспечения (управления) безопасной эксплуатации потенциально опасного оборудования [1 - 3].

Металл элементов энергетического оборудования (таких, как роторы паровых турбин) работает в критических условиях: при высоких температурах и при воздействии высоких напряжений, вызванных усилиями самокомпенсации и массовыми нагрузками.

В практике эксплуатации паровых турбин известны серьезные аварии в результате повреждения роторов. Большое внимание привлекли следующие случаи: авария на тепловой электростанции в США (штат Теннесси, 1974 г.); авария в России на Каширской ГРЭС-4 [4] (октябрь 2002 г.); авария в Украине на одном из энергоблоков Приднепровской ТЭС (2007 г.).

Большой интерес представляет исследование металла разрушенных роторов паровых турбин высокого давления для выявления причин их разрушений и для предотвращения в будущем подобных случаев. В настоящей работе приведены результаты исследований металла фрагмента разрушенного ротора паровой турбины высокого давления (рис. 1) после наработки 293061 ч на ОАО «Западно-Сибирская ТЭЦ» (г. Новокузнецк).

При изучении поверхности разрушения установлено, что излом имеет вид, характерный для многоцикловой усталости. На изломе отчетливо различаются зоны, соответствующие трем стадиям: зарождения разрушения, устойчивого (стабильного) роста усталостной трещины и нестабильного (ускоренного) распространения трещины. Очаг разрушения расположен в зоне технологического паза.

Для экспериментальных исследований был вырезан образец из разрушенного ротора паровой турбины высокого давления в виде диска диам. 290 мм и шириной 50 мм, включающий с одной стороны поверхность излома.

Химический состав исследованного металла был определен на оптико-эмиссионном спектрометре

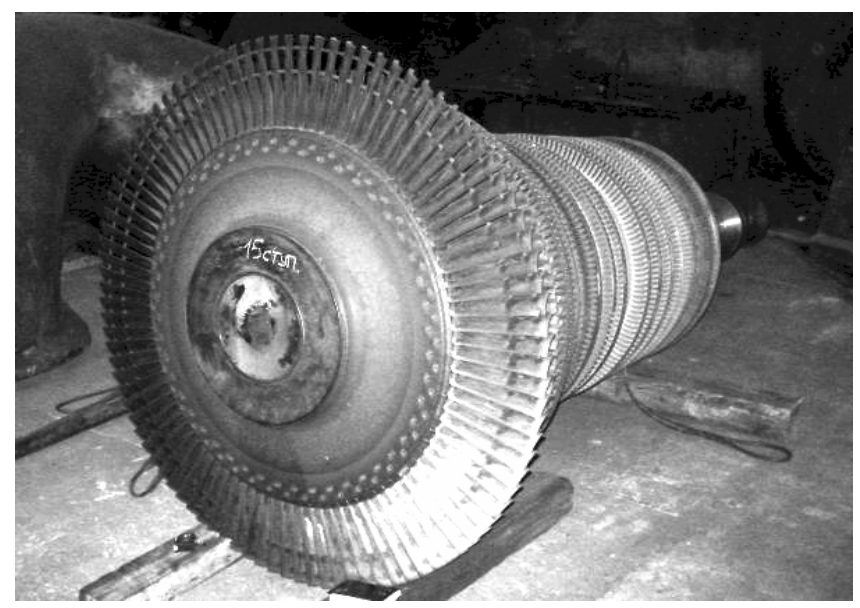

Рис. 1. Общий вид разрушенного ротора паровой турбины высокого давления
«Q4 Tasman». Металл разрушенного ротора содержал $0,34 \% \mathrm{C}, \quad 0,26 \% \mathrm{Si}, \quad 0,37 \% \mathrm{Mn}, \quad 1,53 \% \mathrm{Cr}, \quad 1,6 \% \mathrm{Ni}$, 0,30\% Mo, 0,008 \% P, 0,006 \% S (по массе). Сталь 34ХН$1 \mathrm{MA}$ по ТУ 108-1028 - 81 содержит 0,30-0,40\% C, $0,17-0,34 \% \mathrm{Si}, \quad 0,50-0,80 \% \mathrm{Mn}, \quad 1,30-1,70 \% \mathrm{Cr}$, $1,30-1,70 \% \mathrm{Ni}, 0,20-0,30 \% \mathrm{Mo}, \leq 0,025 \% \mathrm{P}, \leq 0,025 \% \mathrm{~S}$ (по массе).

Ротор паровой турбины изготовлен из стали 34ХН1МА, которая относится к классу конструкционных легированных сталей и предназначена для изготовления валов, роторов, дисков паровых турбин, валов-шестерен, муфт, зубчатых колес и других особо ответственных, тяжело нагруженных деталей, работающих при температуре до $500^{\circ} \mathrm{C}$.

На первом этапе металл разрушенного ротора был исследован спектрально-акустическим методом, который предназначен для изучения структурно-фазового состояния металла и основан на измерении времени задержки $(R, \mathrm{Hc})$ и скорости распространения $(V, \mathrm{M} / \mathrm{c})$ волн Релея (измерительно-вычислительный комплекс «Астрон» [5-9].

Для измерения акустических характеристик исследуемая поверхность образца была разбита на 78 участков. После установки датчика на каждом участке проводили измерения в двух взаимно перпендикулярных направлениях. Для получения достоверных результатов измерения проводили в каждой точке не менее восьми раз, все измерения (скорость и время задержки волны) фиксировали в памяти программного модуля прибора. После проведенных измерений полученные значения обрабатывали методами математической статистики. На поверхности исследуемого диска выявлено пять участков (из 78) со значительными отклонениями акустических характеристик от среднестатистических (рис. 2), из этих участков были вырезаны шлифы для изучения микроструктуры.

Второй этап. В ходе исследования с применением рентгеноструктурного анализа (дифрактометр ДРОН-7) и просвечивающей дифракционной электронной микроскопии на тонких фольгах (просвечивающий элект-

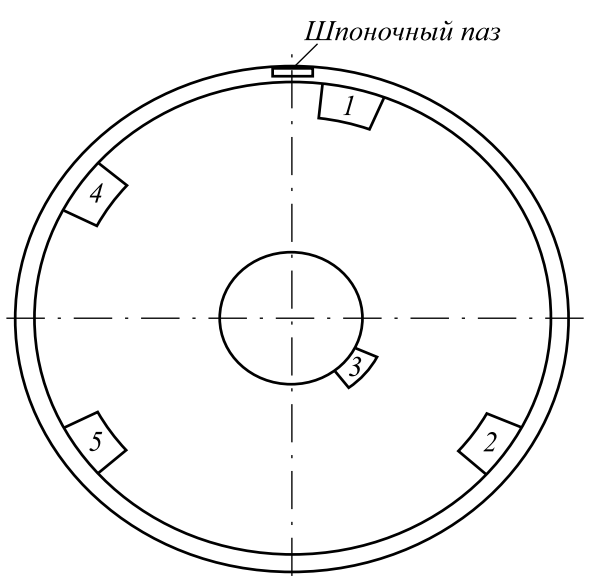

Рис. 2. Схема мест вырезки образцов для исследования 
ронный микроскоп ЭМ-125) [10] установлено, что морфологически структура $\alpha$-фазы во всех исследованных образцах представлена в виде нефрагментированного и фрагментированного бейнита (табл. 1).

За исходное состояние материала была принята структура образца 3 (как наиболее характерная для металла в сечении ротора), которая на $75 \%$ состоит из нижнего бейнита и на $25 \%$ из фрагментированного бейнита (рис. 3).

Амплитуды напряжения сдвига $\sigma$, создаваемого дислокационной структурой, и моментных напряжений $\sigma_{\text {д }}$, создаваемых избыточной плотностью дислокаций, приведены в табл. 2 (амплитуда моментных напряжений

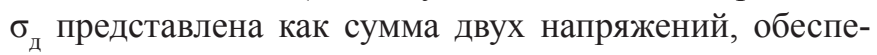
чивающих пластический $\left(\sigma_{\text {пл }}\right)$ и упругий $\left(\sigma_{\text {упр }}\right)$ изгибы кристаллической решетки, т.е. $\sigma_{д}=\sigma_{\text {пл }}+\sigma_{\text {упр }}$ ).

В отличие от исходного образца 3 , в образцах 1,2 , 4 и 5 имеет место упруго-пластический изгиб. В этих образцах сформировались упруго-пластические моментные напряжения, амплитуда которых существенно превышает величину скалывающих (сдвиговых) напряжений $\sigma$. При этом скалывающие напряжения вблизи поверхности излома практически одинаковы, но выше, чем в исходном образце 3 . Амплитуда упругих моментных напряжений практически в два раза меньше, чем пластических. Исключение составляет образец 2, в котором, наоборот, амплитуда упругих моментных напряжений больше пластических практически в три раза.

В структуре образца 2 как в его фрагментированной, так и в нефрагментированной областях наблюдаются микротрещины (рис. 4). Доля материала, занятого областями, которые содержат микротрещины, составляет $25 \%$ (по объему). С одной стороны, образование микротрещин приводит к релаксации напряжений, скалярная плотность в этих областях уменьшается, фрагментированная структура разрушается. С другой стороны, в устье образовавшихся микротрещин присутствуют в виде «факела» тонкие изгибные экстинкционные контуры. Наличие таких контуров свидетельствует о возникновении в этих местах упругих ло-

Т а б ли ц а 1

Содержание морфологических составляющих (вблизи поверхности излома)

\begin{tabular}{|c|c|c|c|}
\hline \multirow{3}{*}{$\begin{array}{c}\text { Обра- } \\
\text { зец }\end{array}$} & \multicolumn{3}{|c|}{ Содержание, \% } \\
\hline & \multirow{2}{*}{$\begin{array}{c}\text { нижний } \\
\text { бейнит }\end{array}$} & \multicolumn{2}{|c|}{ фрагментированный бейнит } \\
\hline & & $\begin{array}{c}\text { анизотропные } \\
\text { фрагменты }\end{array}$ & $\begin{array}{c}\text { изотропные } \\
\text { фрагменты }\end{array}$ \\
\hline 3 & 75 & 25 & 0 \\
\hline 1 & 25 & 60 & 15 \\
\hline 2 & 20 & 80 & 0 \\
\hline 4 & 50 & 50 & 0 \\
\hline 5 & 40 & 40 & 20 \\
\hline
\end{tabular}
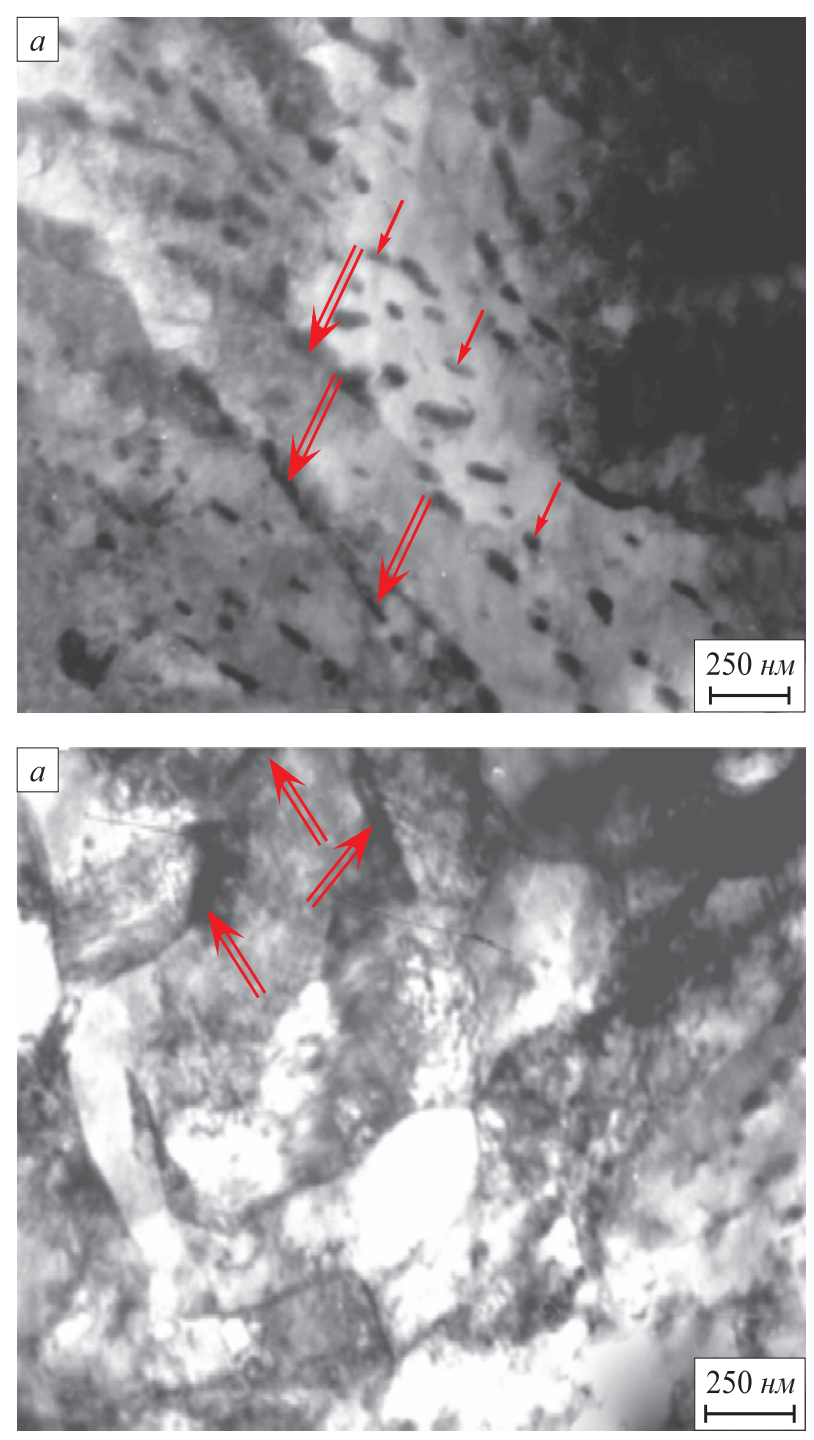

Рис. 3. Электронно-микроскопическое изображение участка образца 3 :

$a$ - нижний бейнит; $\sigma$ - фрагментированный бейнит

Т а б ли ц а 2

Амплитуда напряжения сдвига б и моментных
напряжений $\sigma_{д}$ (числитель и знаменатель) в различных морфологических составляющих структуры и в среднем по материалу

\begin{tabular}{|c|c|c|c|c|}
\hline \multirow{3}{*}{$\begin{array}{l}\text { Обра- } \\
\text { зец }\end{array}$} & \multicolumn{4}{|c|}{ Амплитуды напряжений, МПа } \\
\hline & \multirow{2}{*}{$\begin{array}{l}\text { нижний } \\
\text { бейнит }\end{array}$} & \multicolumn{2}{|c|}{$\begin{array}{c}\text { фрагментированный } \\
\text { бейнит }\end{array}$} & \multirow{2}{*}{$\begin{array}{c}\text { в среднем по } \\
\text { материалу }\end{array}$} \\
\hline & & $\begin{array}{c}\text { анизотроп- } \\
\text { ные фраг- } \\
\text { менты }\end{array}$ & $\begin{array}{c}\text { изотроп- } \\
\text { ные фраг- } \\
\text { менты }\end{array}$ & \\
\hline 3 & $360 / 340$ & $560 / 370$ & $-1-$ & $410 / 350$ \\
\hline 1 & $430 / 370$ & $370 / 370$ & $310 / 310$ & $380 / 360+190$ \\
\hline 2 & $420 / 370$ & $490 / 450$ & $-1-$ & $450 / 420+1100$ \\
\hline 4 & $460 / 340$ & $400 / 400$ & $-1-$ & $430 / 370+150$ \\
\hline 5 & $460 / 340$ & $400 / 400$ & $450 / 450$ & $440 / 390+260$ \\
\hline
\end{tabular}



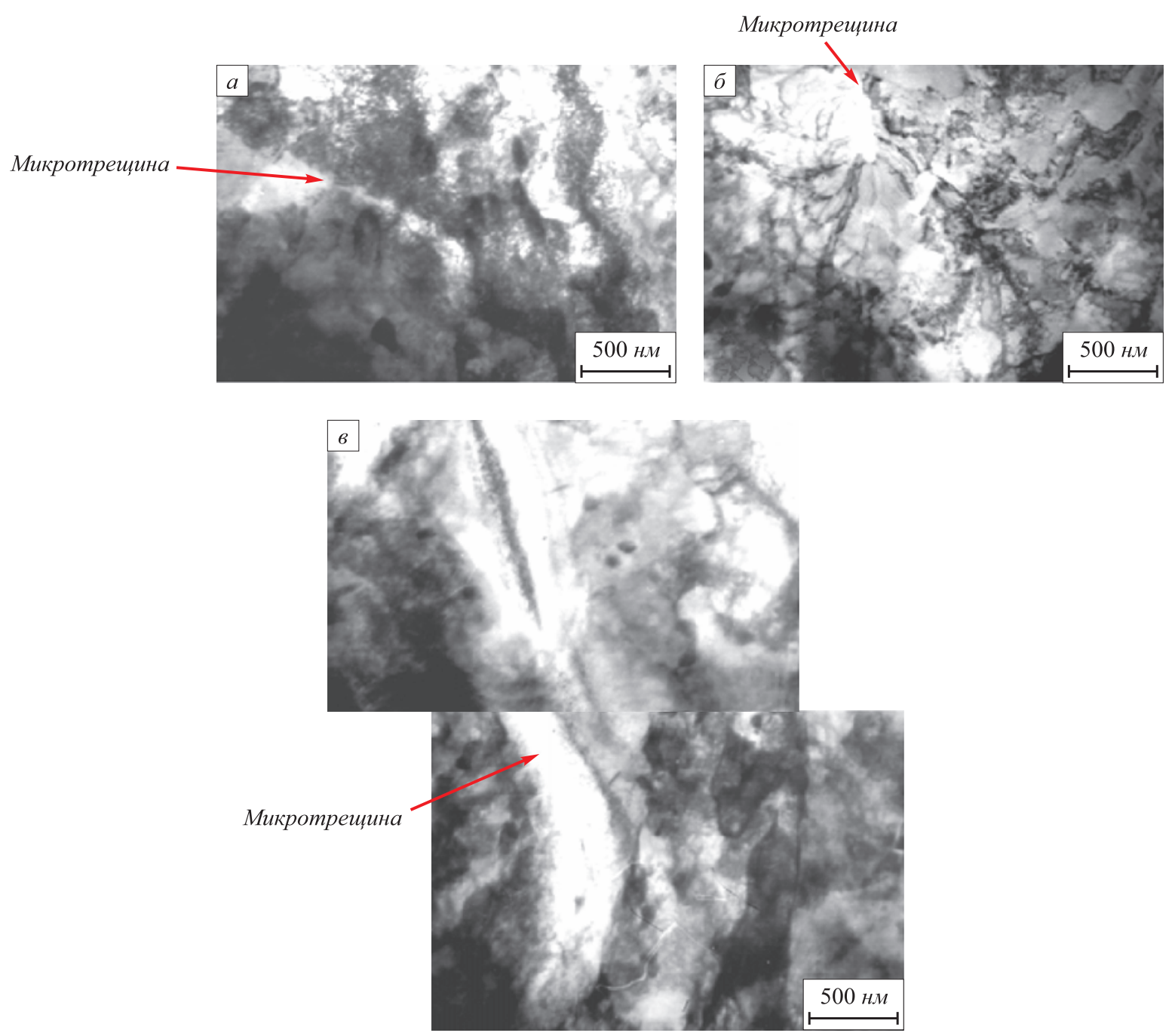

Рис. 4. Образование микротрещин в нижнем бейните $(a, \sigma)$ и фрагментированном бейните с анизотропными фрагментами (в) в образце 2

кальных (моментных) напряжений, амплитуда которых возрастает примерно до 4400 МПа; поэтому амплитуда локальных (моментных) напряжений в образце 2 по сравнению с другими исследованными участками изделия в 2,5 - 3 раза выше.

В итоге было проведено сравнение полученных средних по материалу значений $\sigma$ и $\sigma_{\text {д }}$ р результатами измерения акустических характеристик исследуемого металла. Анализ результатов микроскопических исследований и измерения акустических характеристик показал высокую чувствительность и эффективность спектральноакустического метода (рис. 5) для оценки изменений микроструктуры и полей внутренних напряжений.

Установлено, что в участках фрагмента ротора, в которых более высокие значения $\sigma_{\text {д }}$ (т.е. в которых больший вклад вносит упругая составляющая напряжений) отмечаются более высокие значения $R$ и меньшие $V$. Так, в образце 2 помимо самых высоких значений $\sigma_{д}$ отмечены максимальные значения времени задержки и минимальные значения скорости распространения волн Релея.

Bblвodb. Спектрально-акустическим методом в зоне излома разрушенного ротора выявлены четыре участка с акустическими характеристиками, значительно отличающимися от характеристик на остальных участках сечения ротора (время задержки возрастает до 5839 нс). В отличие от исходного металла, в этих участках (образцах) сечения сформировались упругопластические моментные напряжения, амплитуда кото-

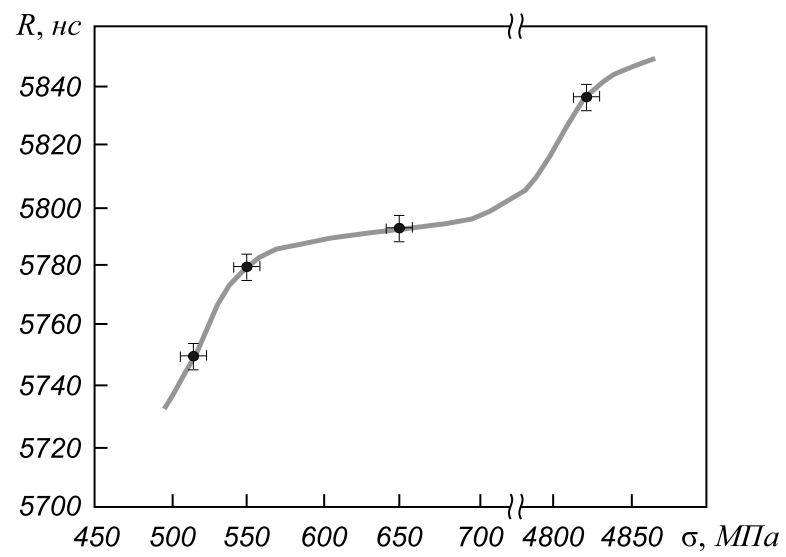

Рис. 5. Зависимость временем задержки ПАВ от среднего по материалу значения моментных напряжений 
рых существенно превышает величину скалывающих (сдвиговых) напряжений, причем амплитуда упругих моментных напряжений почти в два раза меньше, чем пластических. Кроме того, в одном из образцов обнаружены микротрещины, в этом образце амплитуда упругих моментных напряжений больше пластических практически в три раза. Наличие отдельных участков в сечении ротора с высоким уровнем внутренних напряжений (с микротрещинами) способствовало ускорению разрушения. Образование этих участков произошло, вероятнее всего, при изготовлении ротора. Применение спектрально-акустического метода для оценки качества роторов турбин может оказаться эффективным экспресс-методом предотвращения аварийных ситуаций.

\section{БИБЛИОГРАФИЧЕСКИЙ СПИСОК}

1. Резинских В.Ф., Гладштейн В.И., Авруцкий Г.Д. Увеличение ресурса длительно работающих паровых турбин. - М.: изд. МЭИ, 2007. - 296 c.
2. Смирнов А.Н., Абабков Н.В. // Известия Самарского научного центра РАН. 2010. Т. 12. № 1(2). С. 520 - 524.

3. Иванов Ю.Ф., Громов В.Е., Горбунов С.В., Воробьев С.В., Коновалов С.В. // Физика металлов и металловедение. 2011. Т. 112. № 1. C. $85-93$.

4. Загретдинов И.Ш., Костюк А.Г., Трухний А.Д., Должанский П.Р. // Теплоэнергетика. 2004. № 5. С. 5 - 15.

5. Митенков Ф.М., Углов А.Л., Пичков С.Н., Попцов В.М. // Проблемы машиноведения и надежности машин. 1998. № 3. С. 3 - 9 .

6. Углов А.Л., Ерофеев В.И., Смирнов А.Н. Акустический контроль оборудования при изготовлении и эксплуатации. - М.: Наука, 2009. - 278 c.

7. Перевалов С.П., Пермикин В.С., Бархатов Б.В., Гофман Ю.М. // Электрические станции. 1992. № 5. С. 43 - 47.

8. Углов А.Л., Попцов В.М. // Машиностроитель. 1993. № 11. С. 2 - 4.

9. Муравьев В.В, Муравьев М.В., Бехер С.А. // Дефектоскопия. 2002. № 8. С. $53-65$.

10. Смирнов А.Н., Козлов Э.В. Субструктура, внутренние поля напряжений и проблема разрушения паропроводов из стали 12Х1МФ. - Кемерово: Кузбассвузиздат, 2004. - 163 с.

(C) 2014 г. Смирнов А.Н., Абабков Н.В., Козлов Э.В., Конева Н.А., Быкова Н.В Поступила 9 января 2014 г.

Izvestiya VuZov. Chernaya Metallurgiya = Izvestiya - Ferrous Metallurgy. 2014. No. 10. Vol. 57, pp. 67-71.

\section{MICROSTRUCTURE, FIELDS OF INTERNAL STRESSES AND ACOUSTIC CHARACTERISTICS OF METAL OF DESTROYED STEAM TURBINE ROTOR}

\author{
Smirnov A.N. ${ }^{1}$, Dr. Sci. (Eng.), Professor \\ (galvas.kem@gmail.com) \\ Ababkov N..$^{1}$, Cand. Sci. (Eng.), Assist. Professor \\ Kozlov E.V. ${ }^{2}$, Dr. Sci. (Phys.-Math.), Professor, head of De- \\ partment \\ Koneva N.A. ${ }^{2}$, Dr. Sci. (Phys.-Math.), Professor \\ Bykova N.V.1, engineer scientific and innovative management \\ ${ }^{1}$ Kuzbass State Technical University named after T.F. Gorbachev \\ (28,Vesennaya str., Kemerovo, 650000, Russia) \\ ${ }^{2}$ Tomsk State University of Architecture and Civil Engineering \\ (2, Solyanaya sqr., Tomsk, 634003, Russia)
}

Abstract. To identify the causes of destruction and to prevent future similar cases, this paper focuses on research of the metal fragment of destroyed steam turbine rotor by destructive and non-destructive methods. Using methods of electronic microscopy, a metal structure condition of destroyed rotor is researched. The correlation between acoustic characteristic with metal microstructure parameters of destroyed rotor is established.

Keywords: rotor of the steam turbine, microstructure, microcrack, diagnostic.

\section{REFERENCES}

1. Rezinskih V.F., Gladstein V.I., Avrutsky G.D. Uvelichenie resursa dlitel'no rabotayushchikh parovykh turbin [Increased resource of continuously operating steam turbines]. Moscow: MEI, 2007. 296 p. (In Russ.)

2. Smirnov A.N., Ababkov N.V. The integrated approach to assessing the work of energy equipment elements. Izvestiya Sam. scient. Center of RAS. 2010, Vol. 12, no. 1 (2). pp. 520-524. (In Russ.).

3. Ivanov J.F., Gromov V.E., Gorbunov S.V., Vorobiev S.V., Konovalov S.V. Gradient structure-phase states, formed in $08 \mathrm{Cr} 18 \mathrm{Ni} 10 \mathrm{Ti}$ steel by cycle fatigue to the failure. Fizika metallov $i$ metallovedenie. 2011, Vol. 112, no. 1. pp. 85-93. (In Russ.).
4. Zagretdinov I.S., Kostyuk A.G., Truhny A.D., Dolzhansky P.R. Destruction of 300 MW turbine unit of Kashirskaya State District Power Station: causes, consequences and output. Thermal Engineering. 2004, no. 5, pp. 5-15. (In Russ.).

5. Mitenkov F.M., Uglov A.L., Pichkov S.N., Poptsov V.M. On a new material con-trol method of equipment damage of NPP, as well as hardware and software tools for its implementation. Problems of Mechanical Engineering, and the reliability of the machines. 1998, no. 3. pp. 3-9. (In Russ.).

6. Uglov A.L., Erofeev V.I., Smirnov A.N. Akusticheskii kontrol oborudovaniya pri izgotovlenii i ekspluatatsii [Acoustic monitoring of the equipment in manufactur-ing and operating]. Moscow: Nauka, 2009. 278 p. (In Russ.).

7. Perevalov S.P., Permikin B. C., Barhatov B.V., Hoffman Yu.M. Metal damage assessment, operating under high-temperature creep, by the acoustic method. Electric Station. 1992, no. 5. pp. 43-47. (In Russ.).

8. Uglov A.L., Poptsov V.M. The new automated system of NDT strength and reliability of machine elements and structures. $\mathrm{Me}$ chanical Engineering. 1993, no. 11, pp. 2-4. (In Russ.).

9. Murav'ev V.V., Murav'ev M.V., Becher S.A. The new automated system of NDT strength and reliability of machine elements and structures. Defectoscopy. 2002, no. 8, pp. 53-65. (In Russ.).

10. Smirnov A.N., Kozlov E.V. Substruktura, vnutrennie polya napryazhenii i problema razrusheniya paroprovodov iz stali $12 \mathrm{Kh} 1 \mathrm{MF}$ [Substructure, internal stress fields and the problem of the destruction of steel steam pipes of $12 \mathrm{Cr} 1 \mathrm{MoW}$ grade]. Kemerovo: Kuzbassvuzizdat, 2004. 163 p. (In Russ.).

Acknowledgements. The authors are grateful to the staff of the Department of Physics of TSUACE: Popova N.A., Fedorishcheva M.V., Sizonenko E.G., Nikonenko E.L. and Kalashnikov M.P. for the participation in research and Fenster B.R. (JSC "Energoprom-M") for the participation in the production experiments.

Received January 9, 2014 